\title{
BRAF inhibitors: efficacious and tolerable in BRAF-mutant acral and mucosal melanoma
}

\author{
X. BAI ${ }^{\ddagger}$, L. L. MAO ${ }^{\ddagger}$ Z. H. CHI, X. N. SHENG, C. L. CUI, Y. KONG, J. DAI, X. WANG, S. M. LI, B. X. TANG, B. LIAN, L. ZHOU, X. Q. YAN, J. GUO, L. SI* \\ Key Laboratory of Carcinogenesis and Translational Research (Ministry of Education), Department of Renal Cancer and Melanoma, Peking \\ University Cancer Hospital \& Institute, Beijing, China \\ *Correspondence: silu15_silu@126.com \\ Contributed equally to this work.
}

\section{Received November 14, 2016 / Accepted February 1, 2017}

\begin{abstract}
BRAF inhibitors substantially have impressive clinical efficacy in cutaneous melanoma. However, their role in acral and mucosal melanoma remains unclear. Records were reviewed of patients with metastatic or unresectable BRAF-mutant acral and mucosal melanoma hospitalized and administrated BRAF inhibitors during January 2011 and March 2016. Clinical data were collected to determine PFS, ORR, DCR, OS, and safety. Among 28 acral and 12 mucosal melanoma patients treated with BRAF inhibitors, median PFS were 3.6 (95\%CI 3.0-6.4) and 4.4 (95\%CI 0.8-12.7) months, median OS were 6.2 (95\%CI 6.1-12.1) and 8.2 (95\%CI 6.6-19.9) months; ORRs were 38.1\% and 20.0\%, DCRs were $81.0 \%$ and $70.0 \%$ in acral and mucosal melanoma, respectively. BRAF inhibitors were well tolerated. The most common adverse effects (AEs) were cutaneous and hematological. Grade 3/4 AEs were relatively rare. In conclusion, BRAF inhibitors have acceptable efficacy and good tolerance in BRAF mutant acral and mucosal melanoma.
\end{abstract}

Key words: BRAF inhibitor, acral, mucosal, melanoma

Malignant melanoma is one of the most common cancers in the western world among Caucasians. It is estimated to be the fifth and seventh most commonly diagnosed cancer in the USA among men and women in 2016, respectively [1]. However, melanoma is well known for its racial disparity, the age standardized incidences vary more than 45-fold among different ethnics [2]. Besides incidence, histological subtype, primary location, genetic background and prognosis also demonstrate significant disparity among races [3]. For example, unlike Caucasian in Europe and North America, where cutaneous melanoma prevails and about half patients share BRAF (v-Raf murine sarcoma viral oncogene homolog B) mutation [4-6]; in Asia, the most common histological subtypes are acral and mucosal melanoma, within which the rates of BRAF mutation are relatively low, namely varying from $10.3-18.8 \%^{7-11}$ and $0-12.5 \%[7,9,12-15]$ among these 2 subtypes, respectively.

Although BRAF inhibition set a milestone in the treatment of cutaneous melanoma [16,17], situation turns out to be more complicated in the setting of acral and mucosal melanoma. Owing to their relative rarity in Caucasians and their low BRAF $\mathrm{V} 600 \mathrm{E} / \mathrm{K}$ mutation rate, there is still ambiguity with regard to the efficacy of BRAF inhibition in these patients. It remains unclear whether or not patients with BRAF mutant acral and mucosal melanoma would gain similar response from BRAF inhibition as with their cutaneous counterparts. To answer this question, we retrospectively analyzed the clinical data of 28 acral and 12 mucosal BRAF mutant melanoma patients administrated BRAF inhibitors, 6 acral and 6 mucosal BRAF mutant melanoma patients treated with chemotherapy alone in our center from 2011 to 2016.

\section{Patients and methods}

Patients and tumor samples. This study retrospectively analyzed records of patients diagnosed as metastatic or unresectable acral and mucosal melanoma with BRAF mutation hospitalized and administrated BRAF inhibitors or chemotherapy alone during January 2011 and March 2016 in the Department of Renal Cancer \& Melanoma in Peking University Cancer Hospital \& Institute. Tumor sample from primary lesion of each patient was obtained for pathological review, BRAF mutational status confirmation, and CDK4/6 
pathway gene copy number variation (CNV) test. Clinical data including but not limited to age, sex, primary location, stage, survival (followed up until October 2016, or missing of follow up, or death) were collected. The primary endpoints were progression free survival (PFS), objective response rate (ORR), and disease control rate (DCR). The secondary endpoints were overall survival (OS) and safety. The study was approved by Peking University Cancer Hospital \& Institute medical ethics committee, and was conducted following the Declaration of Helsinki Principle.

DNA preparation and BRAF mutation detection. Genomic DNA was extracted from formalin-fixed, paraffinembedded sections using a QIAamp DNA FFPE Tissue Kit (Qiagen, Hilden, Germany). Exons 11 and 15 of BRAF gene were amplified by PCR in at least two separate preparations of genomic DNA in order to detect hotspot mutations, following the steps described earlier $[18,19]$.

CDK4/6 pathway gene $\mathrm{CNV}$ detection. The copy numbers of CDK4, CCND1 (cyclin D1), and P16INK4a genes were detected by Q-PCR. All reagents were purchased from Applied Biosystems Incorporated (ABI): CDK4(HS02225231_CN), P16INK4a (HS03717141_CN), CCND1(HS03803699_CN). Rnase $P$ was taken as a reference gene, non-nucleotide water as negative control, normal skin mixed DNA as normal reference. Q-PCR reaction system: $20 \mu \mathrm{L}$, including TaqMan Genotyping Master Mix $10 \mu \mathrm{L}$, TaqMan Copy Number Target Assay $1 \mu \mathrm{L}$, TaqMan Copy Number Reference Assay (Rnase P) 1 $1 \mu \mathrm{L}$, DNA template, non-nucleotide water. Q-PCR was performed in ABI 7500 Fast real-time PCR system. The results were analyzed in ABI CopyCaller 2.0 software.

Clinical assessment of efficacy of BRAF inhibitors. BRAF inhibitors were administrated according to their recommended (vemurafenib and sorafenib) or investigational dosing (BGB-283), namely vemurafenib $960 \mathrm{mg}$ bid, sorafenib 400mg bid, BGB-283 15mg qd, all orally administrated. Tumor assessment was based on Response Evaluation Criteria in Solid Tumors (RECIST) version 1.1.

Statistical analysis. Categorical data were described as frequencies or percentages, and continuous as means \pm standard deviations or median (range). Survival curves were drawn by the method of Kaplan-Meier survival curve and compared by log-rank test. All statistical analyses were two-sided and $\mathrm{P}<0.05$ was judged as significant. All statistical analyses were carried out via SPSS 20.0 software.

\section{Results}

Demographic and clinical characteristics of the patients. 28 acral and 12 mucosal melanoma patients confirmed of harboring BRAF mutations were administrated BRAF inhibitors (including vemurafenib, sorafenib, and BGB-283 - still in phase I clinical trial); 6 acral and 6 mucosal BRAF-mutant melanoma patients with similar profiles were treated with chemotherapy alone between Jan 2011 and Mar 2016 in the Department of Renal Cancer \& Melanoma in Peking Uni- versity Cancer Hospital \& Institute. Except for 4 mucosal melanoma patients unknown of performance status (PS), the rest 48 patients all had good PS with ECOG $\leq 2$.

Acral melanoma patients treated with BRAF inhibitors. Most patients had 2 (10 patients, 35.7\%), 3 (8 patients,

Table 1. Demographic \& clinical characteristics of BRAF mutant acral melanoma patients

\begin{tabular}{|c|c|c|}
\hline \multirow[t]{2}{*}{ Characteristics } & \multicolumn{2}{|c|}{ No. (\%) } \\
\hline & BRAFi & Chemotherapy \\
\hline \multicolumn{3}{|l|}{ Sex } \\
\hline Male & $11(39.3)$ & $5(16.7)$ \\
\hline Female & $17(60.7)$ & $1(83.3)$ \\
\hline \multicolumn{3}{|l|}{ Age/year } \\
\hline$\geq 60$ & $6(21.4)$ & $0(0.0)$ \\
\hline$<60$ & $22(78.6)$ & $6(100.0)$ \\
\hline \multicolumn{3}{|l|}{ Location } \\
\hline Upper extremity & $3(10.7)$ & $0(0.0)$ \\
\hline Lower extremity & $25(89.3)$ & $6(100.0)$ \\
\hline \multicolumn{3}{|l|}{ Stage } \\
\hline III (Unresectable) & $2(7.1)$ & $0(0.0)$ \\
\hline IV & $26(92.9)$ & $6(100.0)$ \\
\hline \multicolumn{3}{|l|}{ Number of involved organs } \\
\hline$<3$ & $12(42.9)$ & $5(83.3)$ \\
\hline$\geq 3$ & $16(57.1)$ & $1(16.7)$ \\
\hline \multicolumn{3}{|l|}{ BRAF mutation status } \\
\hline V600E & $27(96.4)$ & $6(100.0)$ \\
\hline Others* & $1(3.6)$ & $0(0.0)$ \\
\hline Together with other mutations ${ }^{\#}$ & $1(3.6)$ & $0(0.0)$ \\
\hline CDK4/6 pathway CNV & $(n=12)$ & $(n=2)$ \\
\hline P16 & $9(75.0)$ & $0(0.0)$ \\
\hline CCND1 & $2(16.7)$ & $0(0.0)$ \\
\hline CDK4 & $5(41.7)$ & $0(0.0)$ \\
\hline At least one $\mathrm{CNV}$ & $11(91.7)$ & $0(0.0)$ \\
\hline \multicolumn{3}{|l|}{ ECOG performance status } \\
\hline 0 & $9(32.1)$ & $3(50.0)$ \\
\hline 1 & $16(57.2)$ & $2(33.3)$ \\
\hline 2 & $3(10.7)$ & $1(16.7)$ \\
\hline \multicolumn{3}{|l|}{ M-state at first dosage } \\
\hline M0 & $2(7.1)$ & $0(0.0)$ \\
\hline M1a & $1(3.6)$ & $1(16.7)$ \\
\hline M1b & $0(0.0)$ & $0(0.0)$ \\
\hline M1c & $25(89.3)$ & $5(83.3)$ \\
\hline \multicolumn{3}{|l|}{ LDH at first dosage } \\
\hline Normal & $9(32.1)$ & $4(66.7)$ \\
\hline Above ULN & $16(57.1)$ & $2(33.3)$ \\
\hline Unknown & $3(10.7)$ & $0(0.0)$ \\
\hline \multicolumn{3}{|l|}{ Previous therapies } \\
\hline 0 & $15(53.6)$ & $6(100.0)$ \\
\hline 1 & $11(39.3)$ & $0(0.0)$ \\
\hline 2 & $2(7.1)$ & $0(0.0)$ \\
\hline
\end{tabular}

* One patient carried BRAF K601E mutation; \# BRAF V600E and c-Kit E861K mutation. Legend: ECOG, eastern cooperative oncology group; BRAFi, BRAF inhibitor; LDH, lactate dehydrogenase; ULN, upper limits of normal range. 
28.6\%), 4 (3 patients, $10.7 \%$ ), or 5 (4 patients, $14.3 \%)$ different organs involved. The most common metastatic sites included lymph nodes (75.0\%), lung (67.9\%), bones $(42.9 \%)$, subcutaneous and liver (both $32.1 \%)$, spleen (14.3\%), brain (10.7\%), and muscle (7.1\%). 15/28 patients

Table 2. Demographic \& clinical characteristics of BRAF mutant mucosal melanoma patients

\begin{tabular}{|c|c|c|}
\hline \multirow[t]{2}{*}{ Characteristics } & \multicolumn{2}{|c|}{ No. $(\%)$} \\
\hline & BRAFi & Chemotherapy \\
\hline \multicolumn{3}{|l|}{ Sex } \\
\hline Male & $4(33.3)$ & $3(50.0)$ \\
\hline Female & $8(66.7)$ & $3(50.0)$ \\
\hline \multicolumn{3}{|l|}{ Age/year } \\
\hline$\geq 60$ & $5(41.7)$ & $2(33.3)$ \\
\hline$<60$ & $7(58.3)$ & $4(66.7)$ \\
\hline \multicolumn{3}{|l|}{ Location } \\
\hline Head \& neck & $5(41.7)$ & $1(16.7)$ \\
\hline Gastroesophageal & $1(8.3)$ & $1(16.7)$ \\
\hline Anorectal & $2(16.7)$ & $1(16.7)$ \\
\hline Genitourinary & $4(33.3)$ & $3(50.0)$ \\
\hline \multicolumn{3}{|l|}{ Stage } \\
\hline Local advanced & $0(0.0)$ & $0(0.0)$ \\
\hline Metastatic & $12(100.0)$ & $6(100.0)$ \\
\hline \multicolumn{3}{|l|}{ BRAF mutation status } \\
\hline V600E & $9(75.0)$ & $4(67.7)$ \\
\hline Others & $3(25.0)^{*}$ & $2(33.3)^{\mathrm{s}}$ \\
\hline Together with other mutations & $3(25.0)^{\#}$ & $0(0.0)$ \\
\hline \multicolumn{3}{|l|}{ ECOG performance status } \\
\hline 0 & $5(41.7)$ & $5(83.3)$ \\
\hline 1 & $2(16.7)$ & $1(16.7)$ \\
\hline 2 & $1(8.3)$ & $0(0.0)$ \\
\hline Unknown & $4(33.3)$ & $0(0.0)$ \\
\hline \multicolumn{3}{|l|}{ Location of involved sites } \\
\hline Visceral only & $4(33.3)$ & $1(16.7)$ \\
\hline Visceral \& nonvisceral & $6(50.0)$ & $4(66.7)$ \\
\hline Nonvisceral only & $2(16.7)$ & $1(16.7)$ \\
\hline \multicolumn{3}{|l|}{ Number of involved organs } \\
\hline$<3$ & $9(75.0)$ & $3(50.0)$ \\
\hline$\geq 3$ & $3(25.0)$ & $3(50.0)$ \\
\hline \multicolumn{3}{|l|}{ LDH } \\
\hline Normal & $7(58.3)$ & $6(100.0)$ \\
\hline Above ULN & $5(41.7)$ & $0(0.0)$ \\
\hline \multicolumn{3}{|l|}{ Previous therapies } \\
\hline 0 & $6(50.0)$ & $6(100.0)$ \\
\hline 1 & $2(16.7)$ & $0(0.0)$ \\
\hline 2 & $4(33.3)$ & $0(0.0)$ \\
\hline
\end{tabular}

${ }^{*}$ Included 3 patients carrying BRAF D594G, D594N, and G596D mutations, respectively. ${ }^{\mathrm{s}}$ Included 2 patients harboring BRAF D594G, and G596R mutations, respectively. ${ }^{*}$ Included 3 patients bearing BRAF D594G and C-KIT I571V, BRAF V600E and NRAS (Neuroblastoma RAS Viral Oncogene Homolog) Q61R, BRAF V600E and PDGFR (Platelet Derived Growth Factor Receptor) V824I mutations, respectively. Legend: ECOG, eastern cooperative oncology group; LDH, lactate dehydrogenase; ULN, upper limits of normal range.
(53.6\%) took BRAF inhibitors as their first line therapy (7 took vemurafenib, 6 sorafenib, 1 BGB-283); while 13/25 (46.4\%) had systemic chemotherapy before (11 had one line chemotherapy, while 2 had two lines), which basically included dacarbazine, temozolomide, or taxanes. 5 patients had further systemic anti-tumor therapy after progression from BRAF inhibitors, switching to other BRAF inhibitors \pm MEK inhibitor (2 sorafenib, 1 vemurafenib, 2 dabrafenib + trametinib). Demographic data and clinical characteristics of patients are listed in Table 1.

Acral melanoma patients treated with chemotherapy alone. Patients had 1 (2 patients, 33.3\%), 2 (2 patients, 33.3\%), 3 or 4 (1 patient, respectively, $16.7 \%$ ) different organs involved. The most common metastatic sites included lymph nodes (66.7\%), lung (66.7\%), liver (33.3\%), bones, subcutaneous tissue and pleura (all 16.7\%). No brain metastasis was observed in this subgroup. All patients took chemotherapy as their first line treatment (4 with dacarbazine, 1 temozolomide, 1 nab-paclitaxel). 3 patients had further systemic anti-tumor therapy after disease progression, all switching to paclitaxel. Demographic data and clinical characteristics of patients are listed in Table 1.

Mucosal melanoma patients treated with BRAF inhibitors. Most patients had 1 (2 patients, 16.7\%), 2 (7 patients, $58.3 \%$ ), and 4 (2 patients, $16.7 \%$ ) organs involved. The most common metastatic sites included lymph nodes (66.7\%), lung (41.7\%), liver (25.0\%), bone (25.0\%), local recurrence (25.0\%), and adrenal gland (16.7\%). 1 patient had brain metastasis. 6/12 (50.0\%) took BRAF inhibitors as their first line therapy; while the rest $6 / 12$ had systemic chemotherapy before (2 had one line chemotherapy, while 4 had two lines), which basically included dacarbazine, temozolomide, or taxanes. 3 patients had further systemic anti-tumor therapy after progression from BRAF inhibitors, one with mTOR pathway gene aberration took everolimus, one switched to BGB-283, the third took paclitaxel/carboplatin/bevacizumab. Demographic data and clinical characteristics of patients are listed in Table 2.

Mucosal melanoma patients treated with chemotherapy. Patients had 1 ( 2 patients, 33.3\%), 2 (1 patients, 16.7\%), or 3 (3 patients, 50.0\%) organs involved. The most common metastatic sites included lymph nodes (83.3\%), lung (66.7\%), liver (33.3\%), brain and adnexa (both 16.7\%). 1 patient had brain metastasis. 6/6 (100.0\%) took chemotherapy as their first line therapy ( 3 took dacarbazine and other 3 temozolomide). 1 patient had second line chemotherapy with dacarbazine/ fotemustine after progression. Demographic data and clinical characteristics of patients are listed in Table 2.

Survival of acral melanoma patients. As the most recent analysis carried out on Oct 31, 2016; 3 (10.7\%, 2 taking BGB283,1 sorafenib) of total 28 patients in BRAFi subgroup, and 2 (33.3\%, both taking second line paclitaxel) of total 6 patients in chemotherapy subgroup were still on treatment. Others ceased treatment all because of disease progression, no patient discontinued because of adverse effects. 

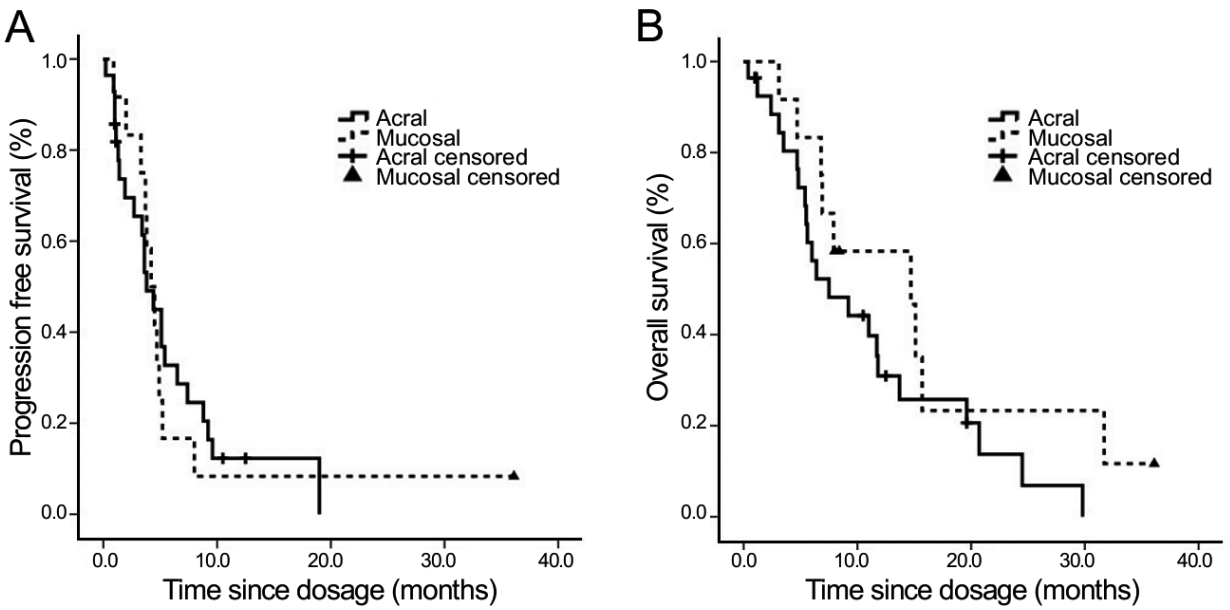

$\begin{array}{lr}\text { Number at risk } \\ \text { Acral } & 28 \\ \text { Mucosal } & 12\end{array}$

\section{Number at risk}

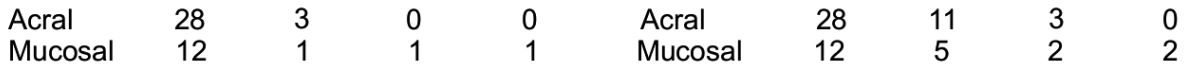

Figure 1. PFS (A) and OS (B) of acral and mucosal melanoma patients. (A) Patients of acral melanoma were shown in solid lines, and mucosal in dotted lines. Tick marks indicated patients censored. Median PFS for acral and mucosal melanoma patients were 3.6 (95\%CI 3.0-6.4) and 4.4 (95\%CI 0.8-12.7) months; (B) and median OS were 6.2 (95\%CI 6.1-12.1) and 8.2 (95\%CI 6.6-19.9) months, respectively. No statistical significant difference regarding survival has been observed between 2 subtypes.

Median PFS for BRAFi and chemotherapy subgroups were 3.6 (95\%CI 3.0-6.4) (Figure 1A) and 2.8 (95\%CI 0.9-3.9) months, respectively. 22 (78.6\%) of 28 patients in BRAFi subgroup and 3 (50.0\%) of 6 patients in chemotherapy subgroup have died, median OS were $6.2(95 \%$ CI 6.1-12.1) months (Figure 1B) and 8.1 (95\%CI 1.1-19.8) months, respectively. Additional follow-up is still going on.

Survival of mucosal melanoma patients. During the last survival analysis, 1 (8.3\%, taking BGB-283) of 12 patients in BRAFi subgroup and non in chemotherapy subgroup was still on treatment; others ceased treatment all because of disease progression, no patients discontinued because of adverse effects. Median PFS for patients treated with BRAFi and chemotherapy were 4.4 (95\%CI 0.8-12.7) (Figure 1A) and 1.1 (95\%CI 0.1-3.3) months, respectively. 9 (75.0\%) of 12 patients in BRAFi and $3(50.0 \%)$ of 6 patients in chemotherapy subgroup have died. Median OS for these two subgroups were 8.2 (95\%CI 6.6-19.9) (Figure 1B) and 4.7 (95\%CI 1.0-9.7) months, respectively. Additional follow-up is still going on.

Response to treatment - acral melanoma. 21/28 in BRAFi and 6/6 in chemotherapy subgroup had documented response data; $18 / 28$ in BRAFi and 2/6 in chemotherapy subgroup had complete radiology data. ORRs were $38.1 \%(8 / 21)$ and $0.0 \%$ (0/6), DCRs were $81.0 \%(17 / 21)$ and $66.7 \%(4 / 6)$ in patients treated with BRAFi and chemotherapy, respectively. Median duration of response was 5.3 (95\%CI 3.8-8.9) months in BRAFi subgroup (Table 3). Among patients with complete radiology data, 14/18 (77.8\%) (Figure 2A) and 1/2 (50.0\%) patients in BRAFi and chemotherapy subgroups had tumor shrinkage, respectively.
Response to treatment - mucosal melanoma. 10/12 patients in BRAFi and 5/6 in chemotherapy subgroup had documented response data; 8/12 in BRAFi and 3/6 in chemotherapy subgroup had complete radiology data. ORRs were $20.0 \%(2 / 10)$ and $0.0 \%(0 / 5)$, DCRs were $70.0 \%(7 / 10)$ and $40.0 \%(2 / 5)$ in BRAFi and chemotherapy subgroup, respectively. Median duration of response was 20.3 months in BRAFi subgroup (table 3 ). Noticeably, only 2 patients had partial response, the duration of response for each was 4.5 and 36.1 months, and the latter had no visceral involvement. In patients with complete radiology data, 4/8 (50.0\%) (Figure 2B) and 2/3 (67.7\%) patients in BRAFi and chemotherapy subgroup had tumor shrinkage, respectively.

Safety issues. Table 4 showed drug-related adverse effects (AEs) reported in at least one patient treated with BRAFi in this study. The most common AEs were cutaneous (handfoot syndrome $14.3 \%$, rash $10.7 \%$, photosensitivity $3.6 \%$ ), hematological (thrombocytopenia 10.7\%, leukopenia 7.1\%,

Table 3. Best response to treatment

\begin{tabular}{lcc}
\hline & Acral $(\mathrm{n}=21)$ & Mucosal $(\mathrm{n}=10)$ \\
\hline Complete response & $0(0.0 \%)$ & $0(0.0 \%)$ \\
Partial response & $8(38.1 \%)$ & $2(20.0 \%)$ \\
Stable disease & $9(42.9 \%)$ & $5(50.0 \%)$ \\
Progressive disease & $4(19.0 \%)$ & $3(30.0 \%)$ \\
Objective response rate $^{*}$ & $8(38.1 \%)$ & $2(20.0 \%)$ \\
Disease control rate $^{*}$ & $17(81.0 \%)$ & $7(70.0 \%)$ \\
\hline
\end{tabular}

${ }^{*}$ Objective response is defined as complete plus partial response. ${ }^{*}$ Disease control was defined as complete response, partial response, and stable disease. 


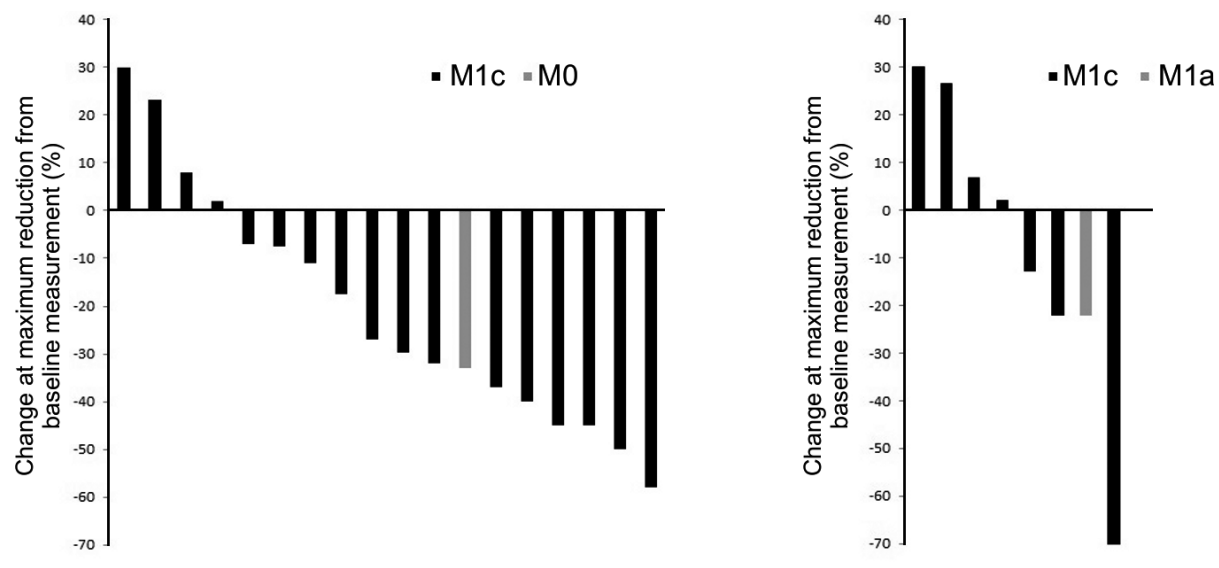

Figure 2. Change at maximum reduction from baseline measurement of acral (A) and mucosal (B) melanoma patients treated with BRAFi. 14/18 (77.8\%) acral and 4/8 (50.0\%) mucosal melanoma patients had tumor shrinkage, median tumor change at maximum reduction from baseline measurement were $-28.3 \%$ and $-5.4 \%$, respectively. $11 / 14(78.6 \%)$ acral and $3 / 4(75.0 \%)$ mucosal patients experienced disease progression after initial tumor shrinkage, median tumor shrinkage lasting time were 5.3 and 6.6 months, respectively.

neutropenia $7.1 \%$, and anemia 3.6\%), arthralgia, pyrexia, fatigue, and alopecia. Grade 3/4 AEs were relatively rare, and the most common one was thrombocytopenia. No secondary skin malignancy was observed. Dose reduction was needed in 3 patients suffering from grade $3 / 4$ thrombocytopenia. No patient discontinued treatment owing to AEs.

\section{Discussion}

Impressive response rates as high as $>50 \%$ have been observed in BRAF mutant cutaneous melanoma treated with

Table 4. Adverse effects of BRAF inhibitors in acral and mucosal melanoma

\begin{tabular}{lccccc}
\hline Adverse effect & \multicolumn{2}{c}{ Acral $(\mathrm{n}=28)$} & & \multicolumn{2}{c}{ Mucosal $(\mathrm{n}=12)$} \\
\cline { 2 - 3 } \cline { 5 - 6 } & $\begin{array}{c}\text { Any grade } \\
(\%)\end{array}$ & $\begin{array}{c}\text { Grade } 3 / 4 \\
(\%)\end{array}$ & & $\begin{array}{c}\text { Any grade } \\
(\%)\end{array}$ & $\begin{array}{c}\text { Grade } 3 / 4 \\
(\%)\end{array}$ \\
\hline Skin & & & & \\
Rash & $3(10.7)$ & $1(3.6)$ & & $1(8.3)$ & $0(0.0)$ \\
Photosensitivity & $1(3.6)$ & $0(0.0)$ & & $0(0.0)$ & $0(0.0)$ \\
Hand-foot syndrome & $4(14.3)$ & $0(0.0)$ & & $0(0.0)$ & $0(0.0)$ \\
Hematological & & & & \\
Leukopenia & $2(7.1)$ & $0(0.0)$ & & $2(16.7)$ & $0(0.0)$ \\
Neutropenia & $2(7.1)$ & $0(0.0)$ & & $1(8.3)$ & $0(0.0)$ \\
Thrombocytopenia & $3(10.7)$ & $2(7.1)$ & & $1(8.3)$ & $1(8.3)$ \\
Anemia & $1(3.6)$ & $0(0.0)$ & & $0(0.0)$ & $0(0.0)$ \\
Others & & & & \\
Arthralgia & $2(7.1)$ & $0(0.0)$ & & $0(0.0)$ & $0(0.0)$ \\
Pyrexia & $2(7.1)$ & $0(0.0)$ & & $1(8.3)$ & $0(0.0)$ \\
Fatigue & $2(7.1)$ & $0(0.0)$ & & $0(0.0)$ & $0(0.0)$ \\
Alopecia & $2(7.1)$ & $0(0.0)$ & & $0(0.0)$ & $0(0.0)$ \\
Dizziness & $1(3.6)$ & $0(0.0)$ & & $0(0.0)$ & $0(0.0)$ \\
Trachyphonia & $1(3.6)$ & $0(0.0)$ & $0(0.0)$ & $0(0.0)$ \\
Proteinuria & $1(3.6)$ & $0(0.0)$ & $0(0.0)$ & $0(0.0)$ \\
\hline
\end{tabular}

BRAF inhibitors $[16,20]$. However, because of its rarity, questions remain whether patients harboring BRAF mutation with acral and mucosal melanoma, the two most common subtypes in Asia, would gain similar benefit from BRAF inhibitors as their counterparts with cutaneous melanoma.

To answer this question, we retrospectively analyzed the data of patients with BRAF mutant acral and mucosal melanoma administrated BRAF inhibitors or chemotherapy alone in our center. Data showed that BRAF inhibitors demonstrated better survival data, higher tumor response rate and disease control rate, compared with chemotherapy. Given the fact that both acral and mucosal melanomas have a worse prognosis compared with cutaneous melanoma [2123 , it is not presumptuous to say that these benefits were substantial. Meanwhile, it is noticeable that the small sample size precluded direct statistical comparison between different treatment subgroups. And the retrospective nature of this study determined that no definitive conclusion could be drawn based on these data. Further prospective studies with larger sample size are needed.

It is note-worthy that compared with cutaneous melanoma, in which the ORR of BRAF inhibitor was above 50\% [16, 17], efficacy of BRAF inhibitors in acral and mucosal melanoma shown in this study (ORR were $38.1 \%$ and $20.0 \%$, respectively) is still less than satisfactory. Similarly, PFS observed in this study were 3.6 and 4.4 months in acral and mucosal melanoma, respectively, verified by Kim HK and colleagues (data unpublished); and shorter than observed in cutaneous melanoma [16, 17].

Three factors may contribute to this phenomenon. The first one is that acral and mucosal melanoma have worse biological behaviors. Second, there may be more co-existing gene mutations in acral and mucosal melanoma, as we found that $11 / 12(91.7 \%)$ acral melanoma patients had CDK4/6 pathway CNV; and as verified by Flaherty KT and colleagues, 
patients with CDK4/6 pathway CNV had shorter PFS and OS when treated with MAPK (mitogen-activated protein kinase) inhibitors compared with CDK4/6 pathway wild type patients [24]. Third, in this study, patients took 3 different kinds of BRAF inhibitors, including vemurafenib, sorafenib, and BGB-283. Sorafenib is a pan-RAF inhibitor, more avidly C-RAF compared with B-RAF. BGB-283 is a BRAF V600 inhibitor in phase I clinical trial now. The efficacy of latter two in BRAF mutant melanoma has not been reported yet. Questions remain on whether or not the mechanism of drug resistance is similar as in cutaneous melanoma [25-27], and whether or not BRAF inhibitors combined MEK inhibitors will yield better clinical outcome too.

A large scale prospective study showed that median OS in metastatic acral and mucosal melanoma patients were 11.4 and 9.1 months, respectively [28]. And in this study, estimated OS for these two subtypes administrated BRAFi were 6.2 and 8.2 months, respectively. However, conclusion about the influence of BRAF inhibitors on OS could not yet be reached, taken the following factors into consideration. First, the ways of calculating OS were different between two studies: one started at the time of first metastasis and the other, BRAF inhibitor dosage. And since in this study 13 acral and 6 mucosal melanoma patients had been previously treated with chemotherapy, the median OS would be considerable longer if calculated in the same way as the first study. Second, it is note-worthy that the first study enrolled BRAF wild type patients, and BRAF mutation is correlated with worse prognosis $[29,30]$. Third, that $89.3 \%$ of acral melanoma patients were $\mathrm{M} 1 \mathrm{c}$ may also contribute to the relative short survival in this study. Prospective matched pair studies of large sample size are required.

Adverse effects were mild and tolerable. The most common AEs were hematological, followed by the ones related to skin. It is suggested to be reasonable to pay more attention to routine complete blood count test during BRAF inhibitor administration. Regular physical examination of skin is also required. Compared with the data from phase 3 prospective randomized clinical trials of vemurafenib, the occurrence of adverse effect in this study was lower ${ }^{17}$. Since the adverse effect data were collected retrospectively, it is anticipated to be under-reported; also, some of the patients in this study took sorafenib or BGB-283 instead of vemurafenib, which would also impact the spectrum of adverse effects.

Future off-the-bench study and prospective clinical trials with larger sample size are required to answer these abovementioned questions.

Acknowledgements: This work was supported by grants from the Major State Basic Research Development Program of China (2013CB911004), National Natural Science Foundation of China (81172196, 81102068, 81272991, 81301984), New Century Excellent Talents in University (NCET-13-0007), Beijing Municipal Natural Science Foundation (7152033) and Beijing Talents Fund (2014000021223ZK26).

\section{References}

[1] SIEGEL RL, MILLER KD, JEMAL A. Cancer statistics, 2016. CA Cancer J Clin 2016; 66: 7-30. https://doi.org/10.3322/ caac. 21332

[2] BRAY F, FERLAY J, LAVERSANNE M, BREWSTER DH, GOMBE MBALAWA C et al. Cancer Incidence in Five Continents: Inclusion criteria, highlights from Volume $\mathrm{X}$ and the global status of cancer registration. Int J Cancer 2015; 137: 2060-2071. https://doi.org/10.1002/ijc.29670

[3] CHI Z, LI S, SHENG X, SI L, CUI C et al. Clinical presentation, histology, and prognoses of malignant melanoma in ethnic Chinese: a study of 522 consecutive cases. BMC Cancer 2011; 11: 85-94. https://doi.org/10.1186/1471-2407-11-85

[4] HALUSKA FG, TSAO H, WU H, HALUSKA FS, LAZAR A et al. Genetic alterations in signaling pathways in melanoma. Clin Cancer Res 2006; 12: 2301s-2307s. https://doi. org/10.1158/1078-0432.CCR-05-2518

[5] LONG GV, MENZIES AM, NAGRIAL AM, HAYDU LE, HAMILTON AL et al. Prognostic and clinicopathologic associations of oncogenic BRAF in metastatic melanoma. J Clin Oncol 2011; 29: 1239-1246. https://doi.org/10.1200/ LCO.2010.32.4327

[6] CURTIN JA, FRIDLYAND J, KAGESHITA T, PATEL HN, BUSAM KJ et al. Distinct sets of genetic alterations in melanoma. N Engl J Med 2005; 353: 2135-2147. https://doi. org/10.1056/NEJMoa050092

[7] SI L, KONG Y, XU X, FLAHERTY KT, SHENG X et al. Prevalence of BRAF V600E mutation in Chinese melanoma patients: Large scale analysis of BRAF and NRAS mutations in a 432-case cohort. Eur J Cancer 2012; 48: 94-100. https:// doi.org/10.1016/j.ejca.2011.06.056

[8] YAMAZAKIN, TANAKA R, TSUTSUMIDA A, NAMIKAWA K, EGUCHI $\mathrm{H}$ et al. BRAF V600 mutations and pathological features in Japanese melanoma patients. Melanoma Res 2015; 25: 9-14. https://doi.org/10.1097/CMR.0000000000000091

[9] GREAVES WO, VERMA S, PATEL KP, DAVIES MA, BAR$\mathrm{KOH}$ BA et al. Frequency and spectrum of BRAF mutations in a retrospective, single-institution study of 1112 cases of melanoma. J Mol Diagn 2013; 15: 220-226. https://doi. org/10.1016/j.jmoldx.2012.10.002

[10] NIU HT, ZHOU QM, WANG F, SHAO Q, GUAN YX et al. Identification of anaplastic lymphoma kinase break points and oncogenic mutation profiles in acral/mucosal melanomas. Pigment Cell Melanoma Res 2013; 26: 646-653. https://doi. org/10.1111/pcmr.12129

[11] VAZQUEZ VDE L, VICENTE AL, CARLONI A, BERARDINELLI G, SOARES P et al. Molecular profiling, including TERT promoter mutations, of acral lentiginous melanomas. Melanoma Res 2016; 26: 93-99. https://doi.org/10.1097/ CMR.0000000000000222

[12] ZEBARY A, JANGARD M, OMHOLT K, RAGNARSSONOLDING B, HANSSON J. KIT, NRAS and BRAF mutations in sinonasal mucosal melanoma: a study of 56 cases. Br J Cancer 2013; 109: 559-564. https://doi.org/10.1038/bjc.2013.373

[13] LYU J, WU Y, LI C, WANG R, SONG H et al. Mutation scanning of BRAF, NRAS, KIT, and GNAQ/GNA11 in oral mucosal 
melanoma: a study of 57 cases. J Oral Pathol Med 2016; 45: 295-301. https://doi.org/10.1111/jop.12358

[14] BUERY RR, SIAR CH, KATASE N, GUNDUZ M, LEFEUVRE $M$ et al. NRAS and BRAF mutation frequency in primary oral mucosal melanoma. Oncol Rep 2011; 26: 783-787. http:// dx.doi.org/ 10.3892/or.2011

[15] PRACHT M, MOGHA A, LESPAGNOL A, FAUTREL A, MOUCHET $N$ et al. Prognostic and predictive values of oncogenic BRAF, NRAS, c-KIT and MITF in cutaneous and mucous melanoma. J Eur Acad Dermatol Venereol 2015; 29: 1530-1538. https://doi.org/10.1111/jdv.12910

[16] HAUSCHILD A, GROB JJ, DEMIDOV LV, JOUARY T, GUTZMER R et al. Dabrafenib in BRAF-mutated metastatic melanoma: a multicentre, open-label, phase 3 randomised controlled trial. Lancet 2012; 380: 358-365. https://doi. org/10.1016/S0140-6736(12)60868-X

[17] MCARTHUR GA, CHAPMAN PB, ROBERT C, LARKIN J, HAANEN JB et al. Safety and efficacy of vemurafenib in BRAF(V600E) and BRAF(V600K) mutation-positive melanoma (BRIM-3): extended follow-up of a phase 3, randomised, open-label study. Lancet Oncol 2014; 15: 323-332. https://doi. org/10.1016/S1470-2045(14)70012-9

[18] BROSE MS, VOLPE P, FELDMAN M, KUMAR M, RISHI I et al. BRAF and RAS mutations in human lung cancer and melanoma. Cancer Res 2002; 62: 6997-7000.

[19] ELLERHORST JA, GREENE VR, EKMEKCIOGLU S, WARNEKE CL, JOHNSON MM et al. Clinical correlates of NRAS and BRAF mutations in primary human melanoma. Clin Cancer Res 2011; 17: 229-235. http://dx.doi. org/10.1158/1078-0432

[20] CHAPMAN PB, HAUSCHILD A, ROBERT C, HAANEN JB, ASCIERTO P et al. Improved survival with vemurafenib in melanoma with BRAF V600E mutation. N Engl J Med 2011;364:2507-2516. https://doi.org/10.1056/NEJMoa1103782

[21] BRADFORD PT, GOLDSTEIN AM, MCMASTER ML, TUCKER MA. Acral lentiginous melanoma: incidence and survival patterns in the United States, 1986-2005. Arch Dermatol 2009; 145: 427-434. http://dx.doi.org/10.1001/archdermatol
[22] CARVAJAL RD, SPENCER SA, LYDIATT W. Mucosal melanoma: a clinically and biologically unique disease entity. J Natl Compr Canc Netw 2012; 10: 345-356. https://doi.org/10.6004/ inccn.2012.0034

[23] MIHAJLOVIC M, VLAJKOVIC S, JOVANOVIC P, STEFANOVIC V. Primary mucosal melanomas: a comprehensive review. Int J Clin Exp Pathol 2012; 5: 739-753.

[24] FLAHERTY KT, DAVIES MA, GROB JJ, LONG GV, NATHAN PD et al. Genomic analysis and $3-y$ efficacy and safety update of COMBI-d: A phase 3 study of dabrafenib (D) + trametinib (T) vs D monotherapy in patients with unresectable or metastatic BRAF V600E/K-mutant cutaneous melanoma. J Clin Oncol 2016; 34: suppl; abstr 9502.

[25] NAZARIAN R, SHI H, WANG Q, KONG X, KOYA RC,et al. Melanomas acquire resistance to B-RAF(V600E) inhibition by RTK or N-RAS upregulation. Nature 2010; 468: 973-977. https://doi.org/10.1038/nature09626

[26] JOHANNESSEN CM, BOEHM JS, KIM SY, THOMAS SR, WARDWELL L et al. COT drives resistance to RAF inhibition through MAP kinase pathway reactivation. Nature 2010; 468: 968-972. https://doi.org/10.1038/nature09627

[27] FEDORENKO IV, PARAISO KH, SMALLEY KS. Acquired and intrinsic BRAF inhibitor resistance in BRAF V600E mutant melanoma. Biochem Pharmacol 2011; 82: 201-209. https://doi.org/10.1016/j.bcp.2011.05.015

[28] KUK D, SHOUSHTARI AN, BARKER CA, PANAGEAS KS, MUNHOZ RR et al. Prognosis of mucosal, uveal, acral, nonacral cutaneous, and unknown primary melanoma from the time of first metastasis. Oncologist 2016; 21: 848-854. https://doi.org/10.1634/theoncologist.2015-0522

[29] NAGORE E, REQUENA C, TRAVES V, GUILLEN C, HAYWARD NK et al. Prognostic value of BRAF mutations in localized cutaneous melanoma. J Am Acad Dermatol 2014; 70: 858-862. https://doi.org/10.1016/j.jaad.2013.10.064

[30] SAFAEE ARDEKANI G, JAFARNEJAD SM, KHOSRAVI S, MARTINKA M, HO V et al. Disease progression and patient survival are significantly influenced by BRAF protein expression in primary melanoma. Br J Dermatol 2013; 169: 320-328. https://doi.org/10.1111/bjd.12351 